Oxygen uptake ( $\mu \mathrm{l}$.) with $40 \mathrm{mgm}$. water-washed, acetone-dried teaoxidase at $p \mathrm{H} 7 \cdot 2$ (phosphate)

\begin{tabular}{|c|rrrrrr|}
\hline Substrate & 10 & 20 & 30 & 40 & 50 & 60 min. \\
\hline 15 mgm. catechol & 37 & 82 & 127 & 175 & 213 & 256 \\
, + + mgm. ascorbic acid & 24 & 53 & 80 & 110 & 135 & 161
\end{tabular}

by the tea-oxidase than catechol. Tyrosinase presumably oxidizes this new polyphenol at a lower rate than catechol, so that in this case an autocatalytic uptake curve is not obtained.

Support for this view is provided by the results observed when ascorbic acid is added to the system. In the presence of ascorbic acid, the rate of uptake is linear and at a significantly lower level than when no ascorbic acid is present. Reduction of the oquinone, immediately on its formation, prevents the formation of the more rapidly oxidized polyphenol, so that the rate observed is that of catechol oxida-
Further details of this work will be published elsewhere later.

E. A. H. Roberts D. J. WooD

Tocklai Experimental Station,

Indian Tea Association, Cinnamara P.O., Assam.

$$
\text { June } 29 .
$$

${ }^{1}$ Wagreich, H., and Nelson, J. M., J. Amer. Chem. Soc., 60, 1545 (1938). ${ }^{2}$ Wright, C. I., and Mason, H. S., J. Biol. Chem., 165, 45 (1946).

\section{Electron Micrograph of a Chromosome of Triton}

DEveloping further the technique employed in our laboratories by Zalokar ${ }^{1}$, we have obtained in our electron microscope (Trüb Täuber, electrostatic) a very large number of pictures of chromosomes of the

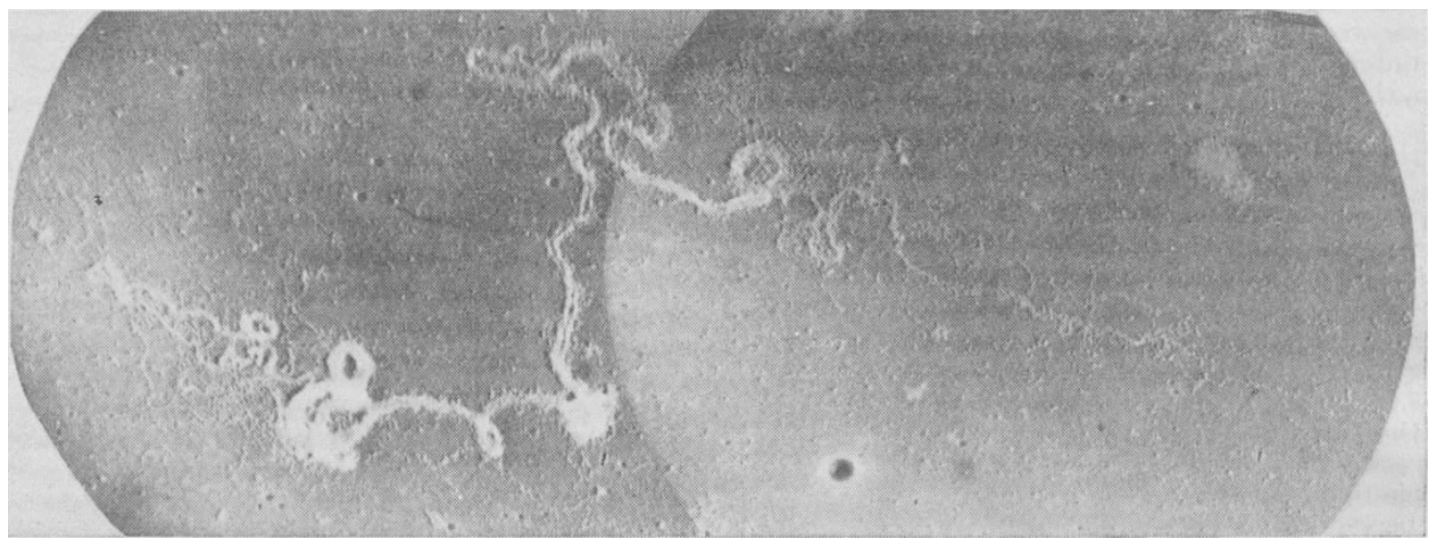

Chromosome (or part of it) centrifuged on collodion membrane, gold-shadowed and enlarged 10,000 times

tion alone, uncomplicated by the formation of a more rapidly oxidized substrate. When all ascorbic acid is oxidized $(3 \mathrm{mgm} .=190 \mu \mathrm{l}$.) the o-quinone is no longer reduced and the reaction-rate then becomes autocatalytic (Figs. 1 and 2).

At $p H$ 7.2 the o-quinone is much less stable than at $p H \mathbf{5 \cdot 6}$, and formation of the new polyphenol is more rapid. Consequently, a slow building up in the rate of oxygen uptake is less likely, and the rate shows only slight acceleration with time. On the other hand, the proportion of the uptake due to oxidation of the second polyphenol is greater in the initial stages, so that ascorbic acid produces a greater inhibition of the rate of uptake, as shown in the accompanying table.

Neither the autocataly tic rate of uptake at $p \mathbf{H} \mathbf{5 \cdot 6}$, nor the depression of the rate of uptake in the presence of ascorbic acid, can be accounted for if catechol is regenerated as a result of the disappearance of $o$ benzquinone from the system. On the other hand, these observations find a complete explanation if, instead of catechol, another polyphenol, oxidized more rapidly by tea-oxidase, is formed as a product of $o$-benzquinone. We cannot at present identify this polyphenol, although there is nothing in our results to exelude the possibility that it may be $1: 2: 4$ trihydroxybenzene. In this case our mechanism differs from that of Wagreich and Nelson only in that the trihydroxybenzene is oxidized enzymically and not by $o$-benzquinone. ovocytes of Triton. The accompanying reproduction shows a typical one. These chromosomes were fixed with acetic acid and 'microcentrifuged'2 on the collodion membrane.

\section{Danon}

Laboratoire de Zoologie,

Laboratoire de Physique, E. GUYÉNOT

\section{E. Kellenberatir}

J. WEIGLE

University of Geneva. July 18.

${ }^{1}$ Dinichert, Guyénot and Zalokar, Rev. Suisse de Zoologie, 54, 283 (1947). ${ }^{2}$ Kellenberger, Experientia, 6. 253 (1949).

\section{Anterior Pituitary Explants of Infantile Rats Grafted in the Anterior Eye Chamber of Hypophysectomized Hosts}

IN an earlier communication ${ }^{1}$, we have shown that hypophysectomized rats, irrespeotive of the length of time during which they were hypophysectomized, responded to pieces of the anterior pituitary introduced in the anterior chamber of the eye by a prompt resumption of growth. Some such grafted pieces of the anterior pituitary survived for periods of sixteen months, showing signs of their activity, as reflected by the growth of the host animal, during all that time. Two animals with sixteen-months old grafts are still alive and still growing. 